This document is published in:

2012 IEEE Conference on Multisensor Fusion and Integration for Intelligent Systems (MFI), pp. 407 - 412.

DOI: 10.1109/MFI.2012.6343070

(C) 2012 IEEE. Personal use of this material is permitted. Permission from IEEE must be obtained for all other uses, in any current or future media, including reprinting/republishing this material for advertising or promotional purposes, creating new collective works, for resale or redistribution to servers or lists, or reuse of any copyrighted component of this work in other works. 


\title{
Context Aided Fusion Procedure for Road Safety Application
}

\author{
Fernando García, Arturo de la Escalera, José María Armingol and Felipe Jiménez
}

\begin{abstract}
Road safety applications require the most reliable and trustable sensors. Context information plays also a key role, adding trustability and allowing the study of the interactions and the danger inherent to them.

Vehicle dynamics, dimensions... can be very useful to avoid misdetections when performing vehicle detection and tracking (fusion levels 0 and 1). Traffic safety information is mandatory for fusion levels 2 and 3 by evaluating the interactions and the danger involved in any detection. All this information is context information that was used in this application to enhance the capacity of the sensors, providing a complete and multilevel fusion application.
\end{abstract}

Present application use three sensors: laser scanner, computer vision and inertial system, the information given by these sensors is completed with context information, providing reliable vehicle detection and danger evaluation. Test results are provided to check the usability of the detection algorithm.

\section{INTRODUCTION}

Recent advances in information technologies are helping to create a wide variety of new applications in numerous fields. One of the fields where these new technologies are becoming ubiquitous is road safety applications. During latest years new applications are helping to prevent danger situations, whether by warning the driver in advance, in case of hazardous situations, or by providing useful information that completes the perception of the environment.

New sensor technologies combined with information technologies are helping to fulfill the strong requirements of road safety applications. But these improvements also lead to more complex applications that have to deal with a wide variety of sensors. In the present application, two of the latest sensors used in road applications are used. Laser scanner and computer vision are combined to provide a fast and robust environment perception, able to overcome the limitations inherent each sensor. First, laser scanner provides robust and reliable obstacle detections but with limited information. Computer vision, in the other hand, provides more information, but less structured and reliable. Thus by combining both sensors these limitations can be overcome. Furthermore, a GPS-inertial system is used to provide information of the movement of the test platform.

* This work was supported by the Spanish Government through the Cicyt projects FEDORA (GRANT TRA2010-20225-C03- 01), Driver Distraction Detector System (GRANT TRA2011-29454-C03-02). CAM through SEGVAUTO (S2009/DPI-1509).

Fernando Garcia, Arturo de la Escalera and José María Armingol are with the Intelligent Systems Lab, Universidad Carlos III of Madrid. \{fegarcia, armingol, escalera\}@ing.uc3m.es.

Felipe Jiménez is with Instituto Universitario de Investigación del Automóvil (INSIA), Universidad Politécnica de Madrid. felipe.jimenez@upm.es.
Besides information from sensors, context information is used to enhance the capacities of the system and to provide complete and multilevel fusion architecture. First, information related to vehicle physics, such as size and dynamics, are used to avoid misdetection, adding reliability. Later, interactions between detections and test platform are checked and the danger that involves them estimated, taking into account vehicle dynamics and traffic safety information.

By the use of the different sensors and context information a multilevel fusion application is obtained. Fig. 1 depicts the overall system including the different data sources.

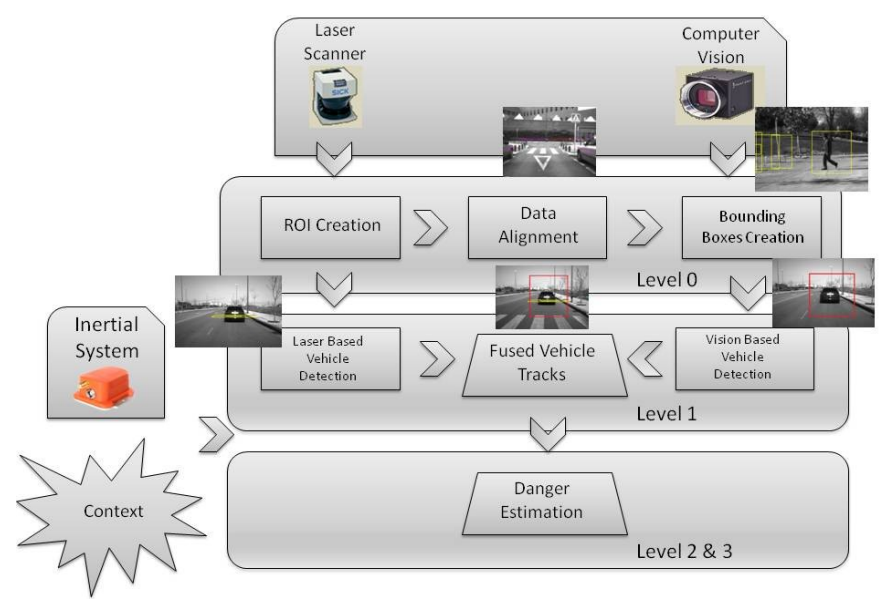

Figure 1. General System architecture

\section{STATE OF THE ART}

Most of the traffic accidents are related with human errors. Distraction and misjudgments are among the most usual causes of these kinds of road events. Taking this into account, recent efforts try to detect these dangerous situations in advance and warn the driver to avoid them. These applications are called Advance Driver Assistant Systems (ADAS).

ADAS applications require reliable sensors that can fulfill the hard requirements that a road safety application demands. Darpa Grand and Urban Challenge proved that laser scanner is one of the key elements for trustworthy road applications ([1], [2], [3] and [4]). Also other recent approaches have proved this [5], [6] and [7].

Laser scanner is a common tool in robotics for Simultaneous Localization and Maping (SLAM) as well as mapping applications. This fact has been translated successfully in several approaches [8], [9], [10], [11], [12], [13] and [14].

Fusion architectures that can be found in transport systems can be divided according to the process architecture used: 
Feature vector fusion. In [15] and [16] features are obtained from each sensor separately and final fusion is performed using different methods: Naïve Bayes, GMMC, NN, FLDA, and SVM.

Dezentralized fusion architectures. In [17] pedestrians are detected using laser scanner and vision. Laser scanner approach is based on multidimensional features, vision algorithm uses Histograms of Oriented Gradients (HOG) features and Support Vector Machine (SVM), final fusion is performed by a Bayesian modeling approach. Similar approach is presented in [16] compared with some feature based models. Finally, [18] performs track to track fusion, using vision and long and short range radars.

A typical configuration when dealing with these two sensors is to use the trustability of laser scanner to give a first region of interest and to perform classification of obstacles based on computer vision. In [19] vehicle detection is performed using this configuration, by using SVM visual classification. In [20] Convolutional Neural Networks is used for pedestrian identification. [21] Uses HOG features and SVM decisor. Finally [22] uses Invariant Features and SVM to identify pedestrians.

\section{SYStem ARChitecture}

The sensors used in the approach are the above mentioned laser scanner, computer vision, as well as a GPS with inertial measurements:

- X-sens MTi-G. It is an accurate GPS aided by an inertial measurement unit. This device can measure not only the velocity of the vehicle, but also some other relevant information for the applications, such as euler angles and angular velocity.

- Laser Scanner from SICK, model LMS-291-S05. Configured to a field of view of 100 degrees, 0.25 degrees of resolution and a maximum distance of 80 meters.

- Point Grade CCD firewire camera, providing images of resolution $640 \times 480$.

Other aspect to take into account of the sensors is the acquisition frequency. The fastest frequency of the system was given by the laser scanner with a period of 52 milliseconds. Thus in order to provide a fast and reliable application, able to respond as fast as possible, the system update time was considered the laser scanner period. The image is received at a different time and it is extrapolated to the time of the next scan of the laser.

Situation awareness is performed, providing fusion solution at levels 2 and 3 . Here context is used to help to calculate two distances, stop distance and safety distance. First is the distance to completely stop the vehicle, thus any obstacle found at shorter distance should be taken into account. Second is the security distance that should be kept between two vehicles to avoid collisions.

Thus the present work proposes a novel method for detecting and classifying vehicles, by using both computer vision and laser scanner. Each sensor performs detection separately, enhanced by the GPS-inertial system, and a final stage combines the detections using a decentralized fusion scheme. Furthermore, the solution given for levels 2 and 3 represent a step forward to provide a multilevel safety application able to fulfill the strong demands of such applications.

\section{Single Sensor Detections}

In this section single sensor detection subsystems are presented. As it is depicted in Fig. 1 laser scanner is used to detect candidates in both sensors subsystems. This way, thanks to the reliability of the laser scanner, false positives in the visual approach are avoided. Thus only in those regions where an obstacle has been found by the laser scanner, vehicle detection is performed. Besides, the computational cost of the process is decreased by reducing the space to search in the image.

\section{A. Laser Scanner vehicle detection}

Laser Scanner detection is based on the algorithm presented in [23]. A brief explanation is included to provide self-content.

Two stages are well differentiated in laser scanner vehicle detection, according to the abstraction level.

First low level detection is performed according to the pattern given by the laser scanner reconstruction. This reconstruction is based in a distance clustering and polyline creation. The pattern searched is shown in Fig. 2.

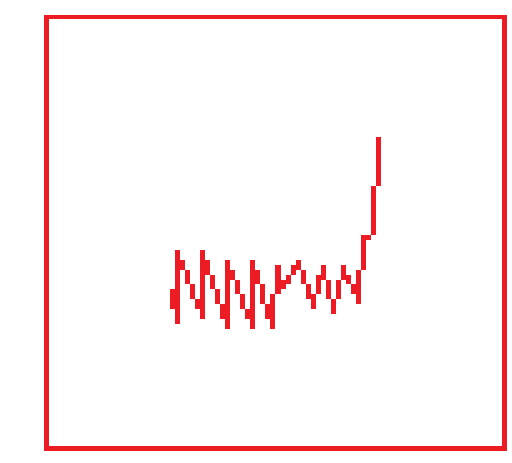

Figure 2. Laser scanner pattern for moving vehicles.

The pattern takes into account the special behavior of the laser scanner, which presents a time delay between the different distances obtained by the laser. Thus for a moving obstacle a common pattern is found. This pattern can also give an estimation of the speed of the vehicle, according to the space between these points and the known delay between them. This way, it is possible to know where it is going to be the detected moving obstacle in the next scan, thus tracking is inherent.

Afterwards, higher level detection correlates the detections with the previous scan detections. At this point higher level classification is performed, based on the movement and the shape of the obstacles. Some constraints are added to avoid false detections, such as impossible movements and velocities.

Context information was important at this level, the constraints used to filter false positives used were based on 
vehicle movement physics: high lateral movements were filtered, as well as impossible accelerations and high dimensional changes.

\section{B. Vision based vehicle detection}

As it has been previously remarked, before performing the camera based detection, Regions Of Interest (ROIs) had to be found. These regions are those spaces in the image where it has been found an obstacle by the laser. The projection of the laser detection in the image is performed and bounding boxes of the region where an obstacle has been found are created (Fig. 3a). Only obstacles with a width big enough to be a vehicle are considered when creating the bounding boxes.

Before creating the bounding boxes, coordinate transformation is mandatory, to transfer the laser scanner coordinate system to camera coordinate system. Finally both sets of data have to be transformed in vehicle coordinate that is referenced as the center of the front bumper of the vehicle (Fig. 4).Equation (1) and (2) depicts the actions performed to change the coordinate systems.

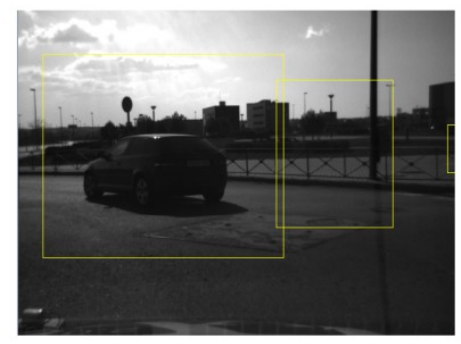

a

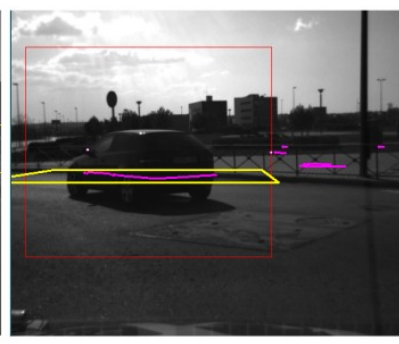

b
Figure 3. Visual vehicle classification. (a) Bounding boxes where the visual algorithm is going to search for the vehicles. (b) Positive vehicle detection (in red) and positive laser scanner detection (in yellow) as well as the laser scanner detection points projected in the image (pink)

$$
\left[\begin{array}{l}
x_{v} \\
y_{v} \\
z_{v}
\end{array}\right]=R_{i}\left(\left[\begin{array}{l}
x_{i} \\
y_{i} \\
z_{i}
\end{array}\right]+T_{i}\right)
$$

where $T_{i}$ is the translation matrix and $R_{i}$ is the rotation matrix that corresponds whether to the laser or the camera $\left(R_{1}\right.$ and $\mathrm{R}_{\mathrm{v}}$ ).

$$
\begin{gathered}
R_{i}=A \cdot B \cdot C \\
A=\left[\begin{array}{ccc}
\cos (\Delta \delta) & 0 & \sin (\Delta \delta) \\
0 & 1 & 0 \\
-\sin (\Delta \delta) & 0 & \cos (\Delta \delta)
\end{array}\right] \\
B=\left[\begin{array}{ccc}
1 & 0 & 1 \\
0 & \cos (\Delta \varphi) & -\sin (\Delta \varphi) \\
0 & \sin (\Delta \varphi) & \cos (\Delta \varphi)
\end{array}\right] \\
C=\left[\begin{array}{ccc}
\cos (\Delta \theta) & -\sin (\Delta \theta) & 0 \\
\sin (\Delta \theta) & \cos (\Delta \theta) & 0 \\
0 & 0 & 1
\end{array}\right]
\end{gathered}
$$

where $\Delta \varphi, \Delta \theta$ and $\Delta \delta$ are the euler angles roll, pitch and yaw respectively between the coordinate axis of each of the reference systems and the vehicle coordinate system.

After changing the coordinate system to camera coordinates, the ROIs have to be transformed to the camera coordinate system $\mathrm{u}, \mathrm{v})$. It is done using the pin-hole model (3).

$$
\lambda\left[\begin{array}{l}
u \\
v \\
1
\end{array}\right]=\left[\begin{array}{llc}
f & 0 & u_{0} \\
0 & f & v_{0} \\
0 & 0 & 1
\end{array}\right]\left[\begin{array}{l}
x_{c} \\
z_{c} \\
y_{c}
\end{array}\right]
$$

where $u_{0}$ and $v_{0}$ are the center coordinates of the camera coordinate system. $\mathrm{u}, \mathrm{v}$ are the coordinates of the camera. And $\mathrm{x}_{\mathrm{c}} \mathrm{y}_{\mathrm{c}}$ and $\mathrm{z}_{\mathrm{c}}$ are the cartesian coordinates of from camera (Fig. 4).

Final classification is performed using Haar Cascade Classifier [24]. In each of the bounding boxes (Fig. 3b).

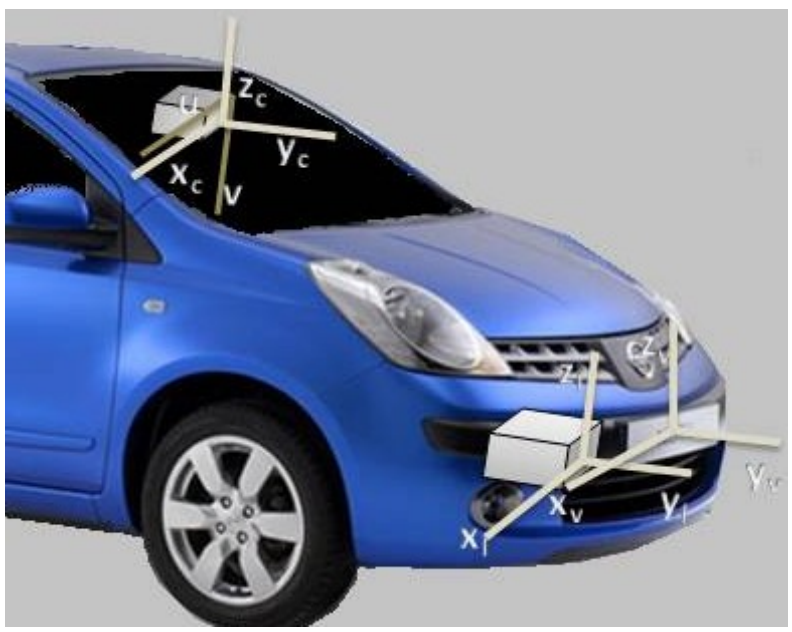

Figure 4. Different coordinate systems $\left(\mathrm{x}_{\mathrm{c}}, \mathrm{y}_{\mathrm{c}}, \mathrm{z}_{\mathrm{c}}\right)$ are camera coordinate system, $\left(\mathrm{x}_{\mathrm{l}}, \mathrm{y}_{\mathrm{l}}, \mathrm{Z}_{\mathrm{l}}\right)$ is the laser coordinate system, and $\left(\mathrm{x}_{\mathrm{v}}, \mathrm{y}_{\mathrm{v}}, \mathrm{Z}_{\mathrm{v}}\right)$ is the coordinate system of the vehicle. Coordinates $(\mathrm{u}, \mathrm{v})$ are the image coordinates.

\section{TRACKING AND DATA ASSOCIATION}

Fusion process at level 1 deals with the tracking problem, including movement estimation, data association, gating and track management. Some aspects were taken into account in the development of the algorithms: information available, the system modeling and the scalability of the system.

\section{A. Tracking}

As it was detailed in section IV, data available consisted in detection coordinates from level 0 . There was more information available from lower levels i.e. width, height, or target velocity based on the laser scanner detections. The low reliability of the velocity measured in the laser scanner subsystem, and the lack of relevance for tracking purpose of the vehicle dimensions made that information not useful. Thus only detection coordinates were considered reliable enough to be included on this stage.

According to the information available and the fast detection frequency, a constant velocity model was considered, modeling the acceleration changes as system errors. This assumes high simplifications that could lead to errors in the tracking procedure, giving that the scope of the applications was centered in interurban roads, where the movements are more stables and predictable these assumptions seems to fulfill the requirements. Further works will deal with the great variety of movements found in urban environments. 
Also the linearity problems that derivates of the use of different technologies with strong nonlinearities (equations (1) to (3)) are solved using an Unscented Kalman Filter.

\section{B. Data Association and Gating}

The data association problem deals with the divergence in the measurements in relation with the predictions. First gating is performed using a square approach (4) .

$$
K_{G l} \sigma_{r}
$$

where $\sigma_{r}$ is the residual standard deviation and $K_{G l}$ is a constant that was empirically chosen.

After data the gating process, data association is performed using normalized distance and stability factor that penalizes the more instable measurements (5). Single correspondence is used

$$
d^{2}=\frac{\left(x_{i}-\bar{x}\right)^{2}}{\sigma_{x}^{2}}+\frac{\left(y_{i}-\bar{y}\right)^{2}}{\sigma_{y}^{2}}+\ln \left(\sigma_{x} \sigma_{y}\right)
$$

\section{Track management}

Two types of tracks were defined, consolidated and non consolidated. First refers to the tracks that were detected by a single sensor, thus the reliability of the track is not high enough to report the vehicle detection, but it is tracked during some scans in case that the other sensor confirms the detection. Consolidated tracks are those that were detected by both subsystems. The track management logic follows the next rules.

- Track creation. It is performed when any of the sensors performs a detection that does not match with any of the existent tracks. Any new detection is considered non-consolidated until both sensors confirm it.

- Track deletion. For a non consolidated track is 3 consecutive scans with no detections, and for a consolidated track 5. First is limited to 3 to penalize them, since they are more likely to be a false positive.

- Track update. Any match of any of the sensors updates a given track.

Table I summarizes the track to track logic.

\section{SITUATION ASSESSMENT AND DANGER ESTIMATION}

Once the obstacles have been detected and tracked, information related to track interactions and the danger involved have to be studied. Here is where context information and GPS-inertial information is important. The second allows to know the current status of our vehicle, such as vehicle velocity, position, etcetera. First is important to know the danger involving any of the detections.

\section{A. Relevant distances}

Distances in road applications are a key issue when dealing with safety. In the present application two distances are relevant.

Braking distance is the distance to completely stop the vehicle. It is important to determine which of the detections in the surroundings of the vehicle should be considered. Other vehicles out of this distance should be tracked, but since the interaction level with the vehicle is limited, the danger that involves these detections is limited.

Safety distance is the distance that it is considered safe in relation to another vehicle that is in front of the vehicle. Among these vehicles those found in a distance shorter that this safety distance should be considered a security thread.

\begin{tabular}{|c|c|c|c|}
\hline $\begin{array}{c}\text { Track vs } \\
\text { New } \\
\text { observation }\end{array}$ & Single sensor & $\begin{array}{c}\text { Both } \\
\text { sensors }\end{array}$ & No match \\
\hline $\begin{array}{l}\text { Non } \\
\text { consolidated }\end{array}$ & $\begin{array}{l}\text { If } \\
\text { sensor } 1=\text { detected } \\
\& \\
\text { sensor } 2=\text { detected } \\
\text { them } \\
\text { track } \\
\text { consolidated. } \\
\text { Otherwise } \\
\text { non consolidated. } \\
\text { Track updated. }\end{array}$ & $\begin{array}{l}\text { Track } \\
\text { consolidated. } \\
\text { Track } \\
\text { updated. }\end{array}$ & $\begin{array}{l}\text { If } \\
\text { \#no detection } \\
>3 \\
\text { them } \\
\text { Track } \\
\text { eliminated. }\end{array}$ \\
\hline Consolidated & Track updated. & $\begin{array}{l}\text { Track } \\
\text { updated. }\end{array}$ & $\begin{array}{l}\text { If } \\
\text { \#no detection } \\
>5 \\
\text { them } \\
\text { Track } \\
\text { eliminated. }\end{array}$ \\
\hline No match & $\begin{array}{l}\text { New } \\
\text { non consolidated } \\
\text { track }\end{array}$ & $\begin{array}{l}\text { New } \\
\text { consolidated } \\
\text { track. }\end{array}$ & \\
\hline
\end{tabular}

TABLE I. TRACK MANAGEMENT SUMMARY

\section{Braking distance}

To calculate braking distance, traffic accident reconstruction math was taken into account [25]. The calculations were based in the worst case scenario, when the vehicle is fully loaded, and only front wheels of the vehicle are blocked. In these situations the friction coefficient applied changes. Equation (7) shows this worst case scenario math that are used to calculate the distance that the vehicle needs to completely stop.

$$
d_{\text {stopping }}=\frac{v^{2}}{\mathrm{\eta} \mu g}
$$

where $\eta$ is the correction factor for friction coefficient depicted in equation (6), $\mu$ the real friction coefficient for the given road, $g$ is the gravity acceleration and $v$ the velocity. This distance is calculated in meters.

$$
\mathrm{\eta}=\frac{b_{2}}{L-h \mu}
$$


where $b_{2}$ is the longitude to from the mass center of the vehicle to the rear axis, $L$ the longitude of the vehicle and $h$ are the high of the mass center of the vehicle.

But the previously presented equation does not take into account the response time for a human being. Response time is commonly assumed to be 0.66 seconds as it was proved in [26] and corroborated in [27]. This is the mean time for a driver to respond to a visual stimulus. It has to be taken into account that there are some aspects that may vary this time, such as physical condition, age and etcetera. Thus the distance that the vehicle needs to completely stop (braking distance) is given by (8).

$$
d_{\text {braking }}=v t_{\text {response }}+d_{\text {stopping }}
$$

\section{Safety distance}

Safety distance is usually a subjective measure which depends, among other things, on the driver situation and road conditions. In [28], [29] and [30] a policy for safety distance in automatic vehicle is presented, this policy can easily be extrapolated to maneuvered vehicles. According to these researches a safe distance $S_{d i}$ is defined as following:

$$
S_{d i}=\lambda_{1}\left(v_{1}^{2}-v_{2}^{2}\right)+\lambda_{2} v_{1}+\lambda_{3}
$$

where $v_{1}$ is the velocity of the vehicle where the system is mounted (in $\mathrm{m} / \mathrm{s}$ ), $\mathrm{v}_{2}$ is the velocity of the target vehicle (in $\mathrm{m} / \mathrm{s}$ ). And $\lambda_{1}, \lambda_{2}$ and $\lambda_{3}$ are the constant that are defined as follows:

$$
\begin{gathered}
\lambda_{1}=\frac{1}{2 a_{\max }} \\
\lambda_{2}=2 T+\frac{2 a_{\max }}{J_{\max }} \\
\lambda_{3}=\left(T+\frac{2 a_{\max }}{J_{\max }}+\frac{2 a_{\text {max }}^{2}}{J_{\text {max }}^{2}}\right) a_{\text {max }} T
\end{gathered}
$$

where $\mathrm{T}$ is the reaction time presented before, $a_{\max }$ is the maximum acceleration and $J_{\max }$ the maximum jerk.

\section{B. Actions to perform}

It is not in the scope of the present application to study which actions to perform once any danger situation is presented. But some aspects should be taken into account:

When a detection is within breaking distance means that the vehicle detected is inside the field of action of the vehicle thus these detection should be considered and their movements monitored.

Also any detection which distance to the vehicle is shorter than the safety distance should be considered a safety thread since it could lead to a collision. An alarm should be triggered, and in certain situations may be necessary to take control of the vehicle to perform avoiding maneuvers.

\section{TEST AND RESUlts}

Several test were performed, most of them in real traffic conditions with several vehicles performing overtaking maneuvers as well as several vehicles being overtaken. In [23] laser scanner vehicle detection algorithm was tested in static conditions, resulting in up to $80 \%$ of positive detections. At closer distances, the results improved, reaching to a $100 \%$ of positive detections. In movement, these results were lower, as it is presented here, and the amount of false positives was extremely high.

New tests performed include the presented fusion procedure that improves the detections performed by laser scanner adding computer vision. Thus the training process for the visual approach focused on avoiding these false detections by thresholding only the results with higher probabilities.

Tests to prove the utility of the system were performed in interurban scenarios with relatively low traffic. The maneuvers performed included overtaking maneuvers and vehicles overtaking the test platform. The results were of a $60 \%$ of camera detection ( $1 \%$ of false positives), $84 \%$ of laser scanner detection (5\% of false positives). The overall

\begin{tabular}{|c|c|}
\hline $\begin{array}{l}\text { Positive Detections of camera based } \\
\text { based detection subsystem }\end{array}$ & $60.13 \%$ \\
\hline $\begin{array}{l}\text { Positive Detections of laser scanner } \\
\text { based detection subsystem }\end{array}$ & $84.09 \%$ \\
\hline $\begin{array}{l}\text { False Positive Detections of camera } \\
\text { based detection subsystem }\end{array}$ & $1.20 \%$ \\
\hline $\begin{array}{l}\text { False Positive Detections of laser } \\
\text { scanner based detection subsystem }\end{array}$ & $5.30 \%$ \\
\hline Overall System Positive Detection & $90.15 \%$ \\
\hline Overall System False Positives & $0.76 \%$ \\
\hline
\end{tabular}
fusion system was able to perform vehicle detection in $90 \%$ of the detections with less that $1 \%$ of false positives. In Fig. 5 some results images are provided. Table II depicts the results:

TABLE II. TEST RESULTS

\section{CONCLUSION AND FUTURE WORKS}

Presented approach has proved that up to a $90 \%$ of positive detections are possible to be accomplished with a limited number of false positives. This way, laser scanner trustability and information provided by camera can obtain results similar to other well known system widely spread such as frequency radars. The present set of sensors have proved to be very useful to detect other road users such as pedestrian with good results [31]. Thus with the same set of sensor a complete road environment detection can be provided.

Future applications will try to deal with urban scenarios, where the amount of obstacles are higher, thus the false positives found is also higher. More accurate vehicle movement information is important, mainly in curves where the reconstruction of the shape of the detected obstacles by the laser scanner is a tough task. This problem is due to the extreme difficulty to measure the movement of the vehicle using the inertial system. Other approaches, such as steering angle detection, should be taken into account to predict the 
movement of the vehicle in these situations and help in the laser scanner shape reconstruction process.
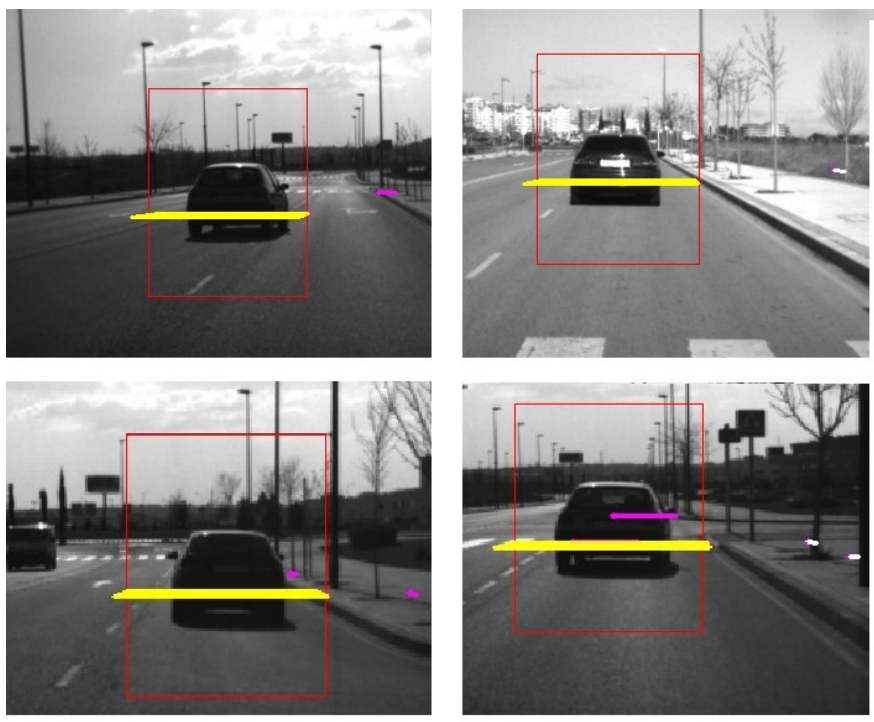

Figure 5. Vehicle detections. In yellow laser scanner positive detection. In red vision based detections.

\section{REFERENCES}

[1] T. Defense and P. Agency, "Foreword for Journal of Field Robotics - Special Issue on the DARPA Grand Challenge," Journal of Field Robotics, vol. 23, no. 9, pp. 657-658, 2006.

[2] K. Iagnemma and M. Buehler Eds, "Special Issue on the DARPA Grand Challenge, Part 1," Journal of Field Robotics, vol. 23, no. 8. pp. 461-652, 2006.

[3] M. Buehler et al., "DARPA Grand Challenge 2005," Industrial Robot An International Journal, vol. 31, no. 5, pp. 1-43-43, 2007.

[4] K. Iagnemma and E. M Buehler, "Special issue on the 2007 DARPA urban challenge," Journal of Field Robotics, vol. 25, pp. 423-860, 2008.

[5] F. Bu and C.-yao Chan, "Pedestrian detection in transit bus application: sensing technologies and safety solutions," IEEE Proceedings Intelligent Vehicles Symposium 2005, vol. 2005, pp. 100-105, 2005.

[6] J. Langheim, A. J. Buchanan, U. Lages, and M. Wahl, "CARSENSE - New environment sensing for advanced driver assistance systems," in Proceedings of the IEEE Intelligent Vehicle Symposium 2001, 2001, pp. 89-94.

[7] D. M. Gavrila, M. Kunert, and U. Lages, "A multi-sensor approach for the protection of vulnerable traffic participants the PROTECTOR project," IMTC 2001 Proceedings of the 18th IEEE Instrumentation and Measurement Technology Conference Rediscovering Measurement in the Age of Informatics Cat No01CH 37188, vol. 3, pp. 2044-2048, 2001.

[8] C.-chih Wang, C. Thorpe, and S. Thrun, "Online simultaneous localization and mapping with detection and tracking of moving objects: theory and results from a ground vehicle in crowded urban areas," 2003 IEEE International Conference on Robotics and Automation Cat NoO3CH37422, vol. 1, pp. 842-849, 2003.

[9] P. Moutarlier and R. Chatila, "Stochastic multisensory data fusion for mobile robot location and environment modeling," 5 th Int Symposium on Robotics Research, vol. 1, no. 2, pp. 85-94, 1989.

[10] F. Nashashibi and M. Devy, "3D Incremental Modeling and Robot Localization in a Structured Environment Using a Laser Range Finder," in ICRA (1), 1993, pp. 20-27.

[11] C. Fruh and A. Zakhor, Fast 3D model generation in urban environments, vol. 54. VDI/VDE Soc. Meas. \& Autom. Control, 2002, pp. 165-170.

[12] H. Zhao and R. Shibasaki, "A vehicle-borne urban 3-D acquisition system using single-row laser range scanners.," IEEE transactions on systems man and cybernetics Part B Cybernetics a publication of the IEEE Systems Man and Cybernetics Society, vol. 33, no. 4, pp. 658-666, 2003

[13] C.-C. Wang, C. Thorpe, and A. Suppe, "Ladar-Based Detection and Tracking of Moving Objects from a Ground Vehicle at High Speed," in Proceedings of the IEEE International Conference on Intelligent Vehicles Symposium IVS, 2003.

[14] C. Thorpe, "A hierarchical object based representation for simultaneous localization and mapping," 2004 IEEERSJ International Conference on Intelligent Robots and Systems IROS IEEE Cat No04CH37566, vol. 1, pp. 412-418, 2004.

[15] C. Premebida, O. Ludwig, M. Silva, and U. Nunes, "A Cascade Classifier applied in Pedestrian Detection using Laser and Imagebased Features," Transportation, pp. 1153-1159, 2010.

C. Premebida, O. Ludwig, and U. Nunes, "LIDAR and VisionBased Pedestrian Detection System," Journal of Field Robotics, vol. 26, no. Iv, pp. 696-711, 2009.

[17] L. Spinello and R. Siegwart, "Human detection using multimodal and multidimensional features," 2008 IEEE International Conference on Robotics and Automation, pp. 3264-3269, 2008.

[18] N. Floudas, A. Polychronopoulos, O. Aycard, J. Burlet, and M. Ahrholdt, "High Level Sensor Data Fusion Approaches For Object Recognition In Road Environment," 2007 IEEE Intelligent Vehicles Symposium, pp. 136-141, 2007.

[19] J. P. Hwang, S. E. Cho, K. J. Ryu, S. Park, and E. Kim, "MultiClassifier Based LIDAR and Camera Fusion," 2007 IEEE Intelligent Transportation Systems Conference, pp. 467-472, 2007.

[20] M. Szarvas and U. Sakai, "Real-time Pedestrian Detection Using LIDAR and Convolutional Neural Networks," in 2006 IEEE Intelligent Vehicles Symposium, 2006, pp. 213-218.

[21] O. Ludwig, C. Premebida, U. Nunes, and R. Ara, "Evaluation of Boosting-SVM and SRM-SVM Cascade Classifiers in Laser and Vision-based Pedestrian Detection," in ITSC, 2011, pp. 15741579

[22] A. Pérez Grassi, V. Frolov, and F. Puente León, "Information fusion to detect and classify pedestrians using invariant features," Information Fusion, vol. 12, no. 4, pp. 284-292, 2010.

[23] F. García et al., "Environment perception based on LIDAR sensors for real road applications," Robotica, vol. FirstView, pp. $1-9,2011$.

[24] P. Viola and M. Jones, "Rapid object detection using a boosted cascade of simple features," Proceedings of the 2001 IEEE Computer Society Conference on Computer Vision and Pattern Recognition CVPR 2001, vol. 1, no. C, p. I-511-I-518, 2001

[25] J. C. Collins, Accident Reconstruction. Charles C. Thomas Publisher, Limited, 1979.

[26] G. Johansson and K. Rumar, "Drivers' brake reaction times.," Human Factors, vol. 13, no. 1, pp. 23-27, 1971.

[27] H. Makishita and K. Matsunaga, "Differences of drivers' reaction times according to age and mental workload.," Accident analysis and prevention, vol. 40, no. 2, pp. 567-575, 2008.

[28] R. E. Fenton, "A headway safety policy for automated highway operations," IEEE Transactions on Vehicular Technology, vol. 28, no. 1, pp. 22-28, 1979.

[29] C. C. Chien and P. Ioannou, "Automatic vehicle-following," in American Control Conference 1992, 1992, pp. 1748-1752.

[30] P. A. Ioannou and C. C. Chien, "Autonomous intelligent cruise control," IEEE Transactions on Vehicular Technology, vol. 42, no. 4, pp. 657-672, 1993.

[31] F. Garcia, A. de la Escalera, J. M. Armingol, J. G. Herrero, and J. Llinas, "Fusion based safety application for pedestrian detection with danger estimation," in Information Fusion (FUSION), 2011 Proceedings of the 14th International Conference on, 2011, pp. 18. 